Research article

\title{
The baboon (Papio anubis) extracranial carotid artery: An anatomical guide for endovascular experimentation J Mocco*1, Daniel J Hoh ${ }^{1}$, M Nathan Nair ${ }^{1}$, Tanvir F Choudhri², William J Mack ${ }^{1}$, Ilya Laufer ${ }^{1}$ and E Sander Connolly Jr${ }^{1}$
}

Address: ${ }^{1}$ Departments of Neurosurgery and Medicine, College of Physicians and Surgeons, Columbia University, New York, USA and 2Department of Neurosurgery, Mount Sinai School of Medicine, New York, USA

E-mail: J Mocco* - jmocco@yahoo.com; Daniel J Hoh - djh32@columbia.edu; M Nathan Nair - mnn6@columbia.edu;

Tanvir F Choudhri - tanvir.choudhri@msnyuhealth.org; William J Mack - wjm32@columbia.edu; Ilya Laufer - ilyalaufter@yahho.com; E Sander Connolly - esc5@columbia.edu

${ }^{*}$ Corresponding author

This article is available from: http://www.biomedcentral.com/|47I-226I///4

(C) 2001 Mocco et al; licensee BioMed Central Ltd. Verbatim copying and redistribution of this article are permitted in any medium for any non-commercial purpose, provided this notice is preserved along with the article's original URL. For commercial use, contact info@biomedcentral.com

\begin{abstract}
Background: As novel endovascular strategies are developed for treating neurological disease, there is an increasing need to evaluate these techniques in relevant preclinical models. The use of non-human primates is especially critical given their structural and physiological homology with humans. In order to conduct primate endovascular studies, a comprehensive understanding of the carotid anatomy is necessary. We therefore performed a detailed examination of the vessel lengths, lumen diameters and angles of origin of the baboon extracranial carotid system.
\end{abstract}

Methods: We characterized the extracranial carotid system often male baboons (Papio anubis, range $15.1-28.4 \mathrm{~kg}$ ) by early post-mortem dissection. Photographic documentation of vessel lengths, lumen diameters, and angles of origin were measured for each segment of the carotid bilaterally.

Results: The common carotid arteries averaged $94.7 \pm 1.7 \mathrm{~mm}$ (left) and $87.1 \pm 1.6 \mathrm{~mm}$ (right) in length. The average minimal common carotid lumen diameters were $3.0 \pm 0.3 \mathrm{~mm}$ (left) and $2.9 \pm$ $0.2 \mathrm{~mm}$ (right). Each animal had a common brachiocephalic artery arising from the aorta which bifurcated into the left common carotid artery and right braciocephalic artery after $21.5 \pm 1.6 \mathrm{~mm}$. The vascular anatomy was found to be consistent among animals despite a wide range of animal weights.

Conclusions: The consistency in the Papio anubis extracranial carotid system may promote the use of this species in the preclinical investigation of neuro-interventional therapies.

\section{Background}

There has been a recent interest in developing aggressive interventional strategies for the treatment of a variety of neurological diseases including stroke, subarachnoid hemorrhage, and head trauma [1-4]. Successful transla- tion of these therapies to the clinical arena, however, is critically dependent on the use of appropriate experimental models [5]. Non-human primate models of neurological diseases currently exist and have the advantage of most closely mimicking human physiology [6]. These 
models are particularly relevant to neuro-interventional research in that anatomical similarities permit routine vascular access and evaluation of devices designed on a clinically relevant scale.

Conducting experimental primate endovascular studies, however, requires a comprehensive understanding of the carotid vascular system. Previous investigations of nonhuman primate vascular anatomy have focused primarily on the general morphology of the vessels and not on vessel angles, lengths, or lumen diameters which are necessary for guiding endovascular technology $[7,8]$. To answer these questions, we performed a study of extensive dissections of the male Papio anubis to determine the dimensions of the carotid artery system and the extent of its variability.

\section{Methods}

All animal care and experimental procedures were approved by the NIH Guide for the Care and Use of Laboratory Animals. Ten male baboons, weighing $19.6 \pm 1.4$ (range: $15.1-28.4$ ) kg, underwent post-mortem examination to determine vessel lengths, lumen diameters, and angles of origin of the extracranial carotid system. At dissection, a sternotomy was performed, the pericardial sac opened, and the arch of the aorta was extensively exposed. The branch vessels arising from the aorta which supply the head, neck, and both upper extremities were further dissected and identified according to homologous structures in humans (Figure 1a). The angles of origin (degrees) were measured for each vessel relative to the parent artery. The length $(\mathrm{mm})$ of each vessel was measured from its origin to its bifurcation into daughter vessels. The extracranial length of bilateral internal carotid arteries was measured from the carotid bifurcation rostrally to the skull base. The greatest lumen diameter of the vessels $(\mathrm{mm})$ was determined by measuring the external vessel width at maximum saline perfusion minus the thickness of the vessel walls in cross section. Dual measurements were performed for relevant arteries with the neck both in flexion and extension.

Two animals underwent contrast magnetic resonance angiography (MRA) just prior to autopsy. Gadolinium (25 cc) was perfused through a peripheral venous line and coronal images of the vessels of interest were generated. The arterial branch anatomy was confirmed radiographically and parallel MRA measurements were performed for comparison with autopsy values.

\section{Results}

Mean measurements with SEM and range are represented (Table 1). A schematic with mean values is provided (Figure 2).

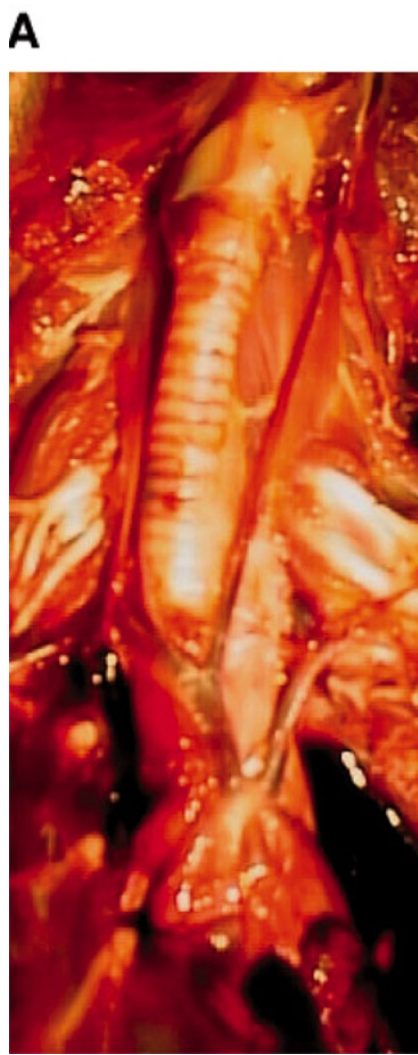

B

\section{Figure I}

Papio anubis extracranial carotid system. The baboon extracranial carotid system was a) dissected at autopsy and b) represented by contrast magnetic resonance angiography.

Contrast MRA scans were obtained for two animals. Radiography revealed the same branch anatomy as observed at autopsy (Figure 1B). The mean differences between MRA and autopsy measurements were $4.6 \pm 1.1$ $\mathrm{mm}$ (length) and $0.9 \pm 0.1 \mathrm{~mm}$ (lumen diameter). The mean difference in angle of origin radiographically and at autopsy was $22.6 \pm 6.0^{\circ}$.

\section{Discussion}

Developing technology for innovative neuro-interventional therapies requires appropriate experimental models for safety, feasibility, and efficacy studies. The use of non-human primates in such preclinical investigations offers significant benefits. Primates share closer structural and physiological homology when compared to other animal species. Also, conventional endovascular techniques in adult baboons may be performed safely and easily when compared to smaller animals with limited vascular access. Conducting primate endovascular studies, however, is dependent on a comprehensive understanding of the anatomy of the baboon extracranial carotid system and the extent of its variability. Our study 


\section{Right Carotid}

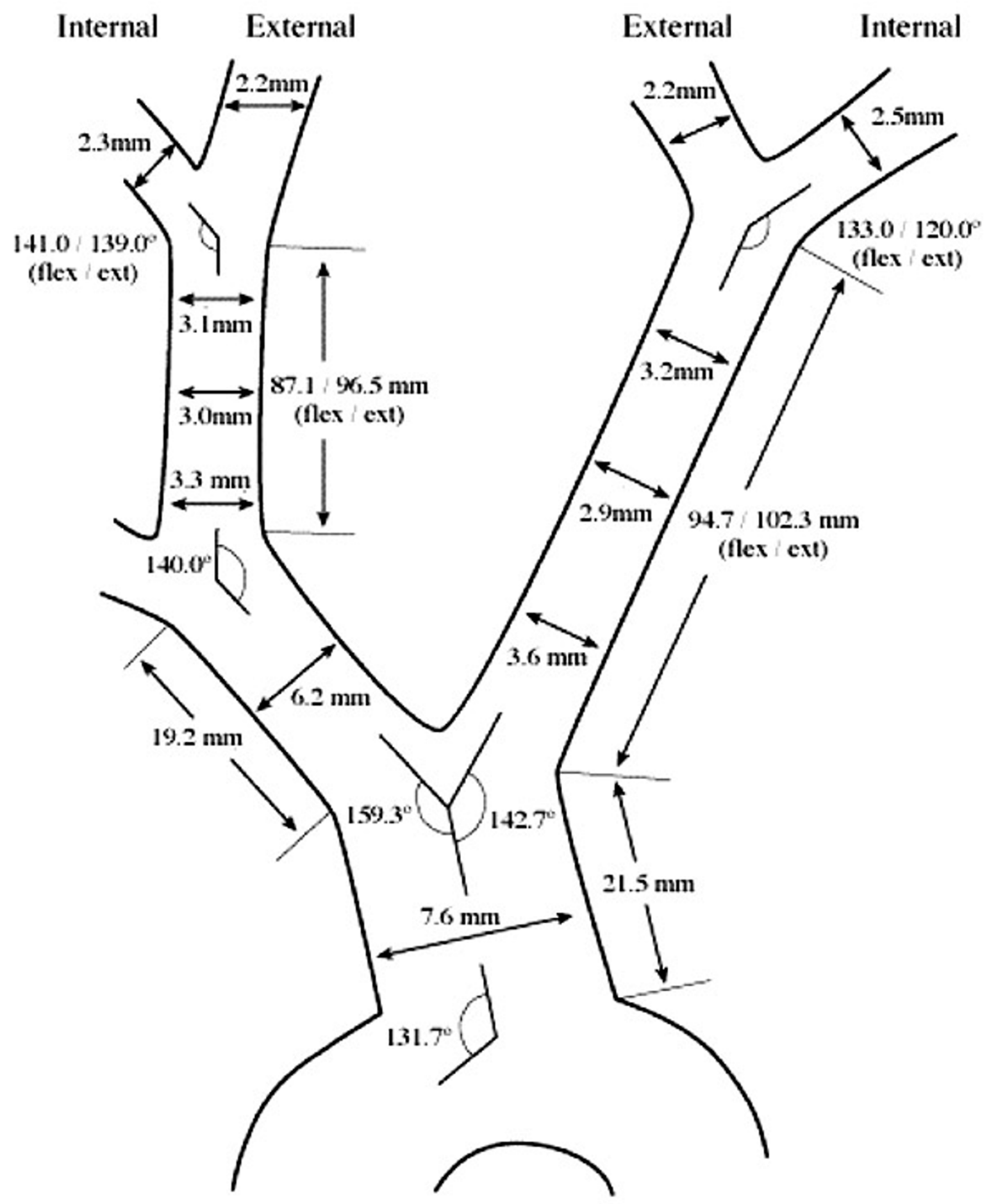

Figure 2

Schematic of Papio anubis anatomy with mean values. Mean vessel lengths $(\mathrm{mm})$, lumen diameters ( $\mathrm{mm})$, and angles of origin (degrees) are represented for the baboon extracranial carotid system. 
Table I: Dimensions of Papio anubis extracranial carotid artery anatomy.

\begin{tabular}{|c|c|c|}
\hline Characteristc & Mean \pm SEM & Range \\
\hline Weight (kg) & $19.6 \pm 1.4$ & $(15.1-28.4)$ \\
\hline \multicolumn{3}{|l|}{ Artery Length (mm) } \\
\hline Common Brachiocephalic & $21.5 \pm 1.6$ & $(10.0-30.0)$ \\
\hline Right Brachiocephalic & $19.2 \pm 1.9$ & $(\mid 3.0-31.0)$ \\
\hline \multicolumn{3}{|l|}{ Left Common Carotid } \\
\hline Neck Flexion & $94.7 \pm 1.7$ & $(90.0-107.0)$ \\
\hline Neck Extension & $102.3 \pm 2.3$ & $(93.0-116.0)$ \\
\hline \multicolumn{3}{|l|}{ Right Common Carotid } \\
\hline Neck Flexion & $87.1 \pm 1.6$ & $(80.0-95.0)$ \\
\hline Neck Extension & $96.5 \pm 1.5$ & $(90.0-102.0)$ \\
\hline Left Internal Carotid & $19.0 \pm 3.1$ & $(6.0-30.0)$ \\
\hline Right Internal Carotid & $19.9 \pm 2.9$ & $(10.0-30.0)$ \\
\hline \multicolumn{3}{|l|}{ Lumenal Diameter (mm) } \\
\hline Common Brachiocephalic & $7.6 \pm 1.0$ & $(4.5-15.0)$ \\
\hline Right Brachiocephalic & $6.2 \pm 0.8$ & $(3.0-11.0)$ \\
\hline \multicolumn{3}{|l|}{ Left Common Carotid } \\
\hline Origin & $3.3 \pm 0.3$ & $(2.0-5.0)$ \\
\hline Middle & $3.0 \pm 0.3$ & $(1.3-4.0)$ \\
\hline Bifurcation & $3.1 \pm 0.3$ & $(2.0-5.0)$ \\
\hline \multicolumn{3}{|l|}{ Right Common Carotid } \\
\hline Origin & $3.6 \pm 0.5$ & $(1.5-6.0)$ \\
\hline Middle & $2.9 \pm 0.2$ & $(2.0-4.0)$ \\
\hline Bifurcation & $3.2 \pm 0.3$ & $(2.0-5.0)$ \\
\hline Left Internal Carotid & $2.5 \pm 0.4$ & $(1.0-5.0)$ \\
\hline Left External Carotid & $2.2 \pm 0.3$ & $(1.0-3.5)$ \\
\hline Right Internal Carotid & $2.3 \pm 0.4$ & $(1.0-4.0)$ \\
\hline Right External Carotid & $2.2 \pm 0.4$ & $(1.0-4.5)$ \\
\hline \multicolumn{3}{|l|}{ Angle of Origin (degrees) } \\
\hline Common Brachiocephalic & $|3| .7 \pm 5.9$ & $(100-155)$ \\
\hline Right Brachiocephalic & $159.3 \pm 2.5$ & $(150-170)$ \\
\hline Left Common Carotid & $142.7 \pm 2.2$ & $(130-152)$ \\
\hline Right Common Carotid & $140.0 \pm 6.8$ & $(100-170)$ \\
\hline \multicolumn{3}{|l|}{ Left Internal Carotid } \\
\hline Neck Flexion* ${ }^{*}$ & $133.0 \pm 6.0$ & $(110-145)$ \\
\hline Neck Extension* & $120.0 \pm 6.3$ & $(90-130)$ \\
\hline \multicolumn{3}{|l|}{ Right Internal Carotid } \\
\hline Neck Flexion* & $140.8 \pm 9.2$ & $(110-170)$ \\
\hline Neck Extension* & $139.2 \pm 8.2$ & $(115-170)$ \\
\hline
\end{tabular}

Dimensions of Papio anubis extracranial carotid artery anatomy. Vessel lengths $(\mathrm{mm})$, lumen diameters $(\mathrm{mm})$, and angles of origin (degrees) were measured at autopsy. Mean values with SEM and range are represented. Dual measurements with the neck in flexion and extension were performed for relevant vessels. Ten subjects were studied for each measurement except where indicated ( $\left.{ }^{*} n=6\right)$.

provides a detailed examination of vessel lengths, lumen diameters, and angles of origin in ten male baboons.

Our findings reveal several important conclusions. First, the species investigated showed little variability in aortic and carotid branch anatomy. All ten animals demonstrated a common brachiocephalic artery giving rise to a left common carotid and right brachiocephalic artery. Second, vessel lengths and lumen diameters were consistent across all animals.
The angle of origin varied considerably for all vessels. This variability may be attributed to several factors. Minor differences in the lengths of parent and daughter vessels may significantly alter the magnitude of the angle of origin. Structural differences in the surrounding soft tissue may also affect the course of branching vessels. Lastly, the degree of neck flexion and extension likely is reflected in the measurements.

Notably, the measurements of vessel lengths and lumen diameters recorded at autopsy were comparable to those 
performed on MRA. MRA, albeit a compiled two-dimensional image, provides a valuable radiographic correlate to the pathology findings. The lumen diameters depicted on MRA specifically represent the vessels at normal physiologic arterial pressure. Interestingly, in the small carotid vessels, the lumen diameters on MRA were measured to be larger than the diameters observed at autopsy. This phenomenon is likely secondary to increased perfusion of smaller vessels in vivo. In any event, these data present a practical lumen diameter to guide endovascular catheter design.

\section{Conclusions}

In conclusion, we found consistent dimensions and little variability in the Papio anubis extracranial carotid system. The uniformity in vessel anatomy and the close homology of baboons to humans suggest that the use of this species for translational models may greatly serve future development of novel endovascular technology.

\section{Competing interests}

None declared

\section{Acknowledgements}

Dr. J Mocco is supported by a Postdoctoral Fellowship from the American Heart Association, Heritage Affiliate. Dr. E. Sander Connolly is supported by the National Institutes of Health (ROI NS 40409). The authors would like to thank Daniel Batista for his excellent technical assistance.

\section{References}

I. Phillips P: New pharmaceutical approaches to stroke prevention, treatment. JAMA I 999, 28 I(22):2075-2076

2. Philips MF, Bagley LJ, Sinson GP, et al: Endovascular thrombolysis for symptomatic cerebral venous thrombosis. J Neurosurg 1999, 90(1):65-7|

3. Kuether TA, Nesbit GM, Barnwell SL: Other endovascular treatment strategies for acute ischemic stroke. Neuroimaging Clin $N$ Amer 1999, 9(3):509-525

4. Fisher M, Bogousslavsky J: Evolving toward effective therapy for acute ischemic stroke. JAMA 1993, 270:360-364

5. del Zoppo G]: Why do all drugs work in animals but none in stroke patients? Drugs promoting cerebral blood flow. I Intern Med 1995, 237:79-88

6. Huang J, Mocco J, Choudhri TF, Poisik A, Popilskis SJ, Emerson R, DelaPaz RL, Khandji AG, Pinsky DJ, Connolly ES: A modified transorbital baboon model of reperfused stroke. Stroke 2000, 3 I:3054-3063

7. Liu X, Branston NM, Kawauchi M, et al: A model of acute focal ischemia in the territory of the anterior cerebral artery in baboons. Stroke 1992, 23(1):40-44

8. Watanabe O, Bremer AM, West CR: Experimental regional cerebral ischemia in the middle cerebral artery territory in primates. Stroke 1977, 8:61-70
Publish with BioMed Central and every scientist can read your work free of charge

"BioMedcentral will be the most significant development for disseminating the results of biomedical research in our lifetime."

Paul Nurse, Director-General, Imperial Cancer Research Fund

Publish with BMC and your research papers will be:

- available free of charge to the entire biomedical community

- peer reviewed and published immediately upon acceptance

- cited in PubMed and archived on PubMed Central

- yours - you keep the copyright 